\title{
Identification of group B streptococci in tissue sections using the peroxidase-antiperoxidase method: a retrospective necropsy study*
}

\author{
T ANDRES AND B MACPHERSON
}

From the Department of Pathology, University of Vermont College of Medicine, Burlington, Vermont 05405, USA

SUMMARY The peroxidase-antiperoxidase (PAP) method was used to demonstrate group B streptococci in formalin-fixed, paraffin-embedded tissue sections using commercial group specific streptococcal antiserum. Sections from neonatal necropsies in which postmortem lung cultures were positive for group B streptococci showed positively stained intra- and extra-cellular cocci in the alveolar exudate. Many hyaline membranes contained intact organisms whereas some membranes showed only diffuse positive staining. One case of neonatal death was evaluated in which there was a clinical diagnosis of group B streptococcal infection, but postmortem cultures were not obtained. Group B streptococci were identified in the lung by the PAP method. This method may be of assistance in the postmortem evaluation of certain infectious diseases such as those due to group B streptococci.

The increasingly frequent recognition of neonatal infections caused by group B $\beta$-haemolytic streptococci has established this microorganism as an important cause of neonatal morbidity and mortality. ${ }^{1-3}$ Despite early diagnosis and the institution of antibiotic therapy the mortality rate remains high, death frequently occurring within 48-72 hours of birth. Antemortem culture results are often incomplete at the time of neonatal death, and the postmortem evaluation of infectious diseases is often fraught with difficulty. Reliable cultures are neither easily obtained nor interpreted. ${ }^{4-8}$ We describe an immunoperoxidase method that can be used to demonstrate group B streptococci in routinely processed paraffin-embedded tissue sections. This technique was used to study 13 neonatal necropsies retrospectively.

\section{Material and methods}

CASE MATERIAL

Necropsies performed at the Medical Center Hospital of Vermont during 1977 and 1978 were reviewed.

*Presented at the Scientific Assembly of the American Society of Clinical Pathologists in Atlanta, Georgia, 24 March 1980.

Received for publication 16 April 1980
Four cases of neonatal death in which postmortem cultures were positive for group B streptococci were selected for investigation. In a fifth case, a presumptive diagnosis of group B streptococcal pneumonitis was made based on the results of antemortem blood cultures and a postmortem tissue Gram stain of lung sections showing Gram-positive cocci.

Eight additional neonatal necropsies were selected for study in which neonatal death had been associated with either pneumonitis or chorioamnionitis, but infection with group B streptococci was not considered likely.

\section{IMMUNOPEROXIDASE METHOD}

The immunoperoxidase (PAP) method was performed essentially as described by Sternberger, ${ }^{9}$ as modified by Taylor. ${ }^{10}$ Sections were made from paraffin-embedded tissues obtained at necropsy. The sections were deparaffinised and placed in a bath of methanol-peroxide to block endogenous tissue peroxidase activity. Tris- $\mathrm{HCl}$ buffer, $\mathrm{pH} \mathrm{7.6,} \mathrm{0.05} \mathrm{M}$, was used throughout the procedure. Specific streptococcal grouping sera prepared in rabbits were obtained from Wellcome Reagents Limited (Wellcome Research Laboratories, Beckenham BR33 BS, UK). These sera are used routinely for the determination of Lancefield groups by the precipitin reactions. Swine anti-rabbit IgG and soluble immune com- 


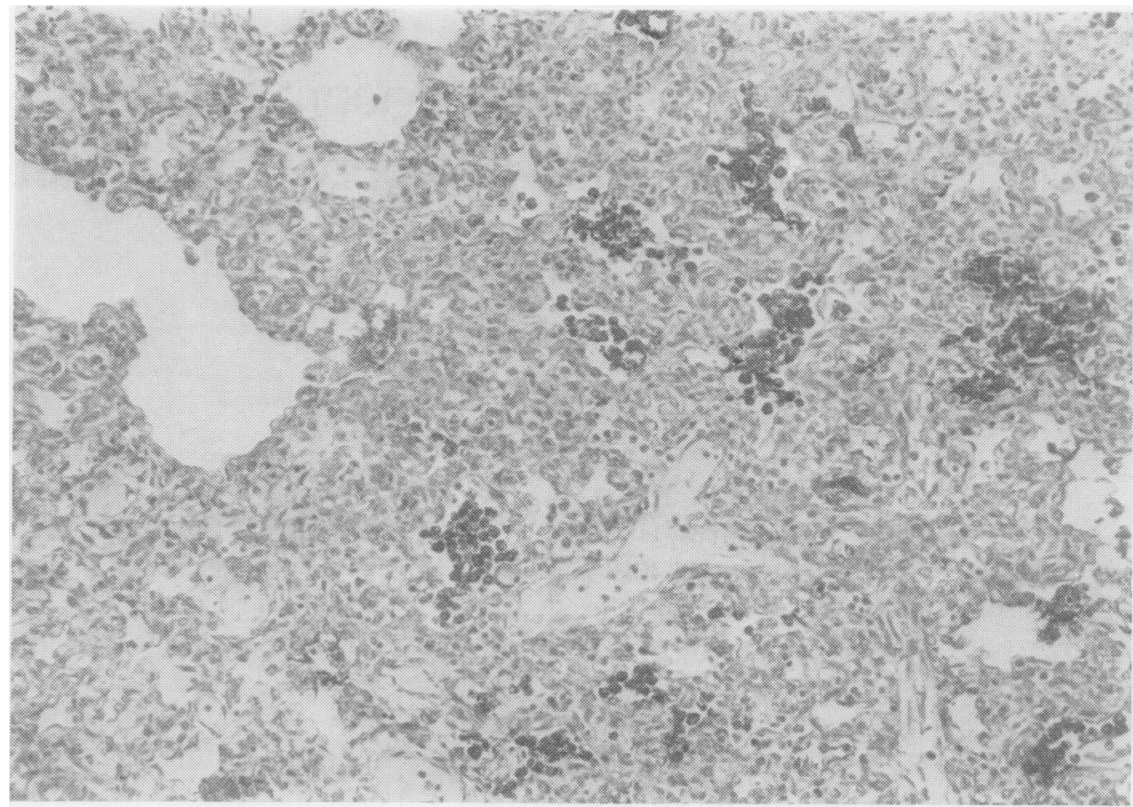

Fig. 1 (a) Neonatal lung with prominent dark staining polymorphonuclear leucocytes within bronchial lumens and alveolar spaces. ( $\times 160$, PAP stain $)$

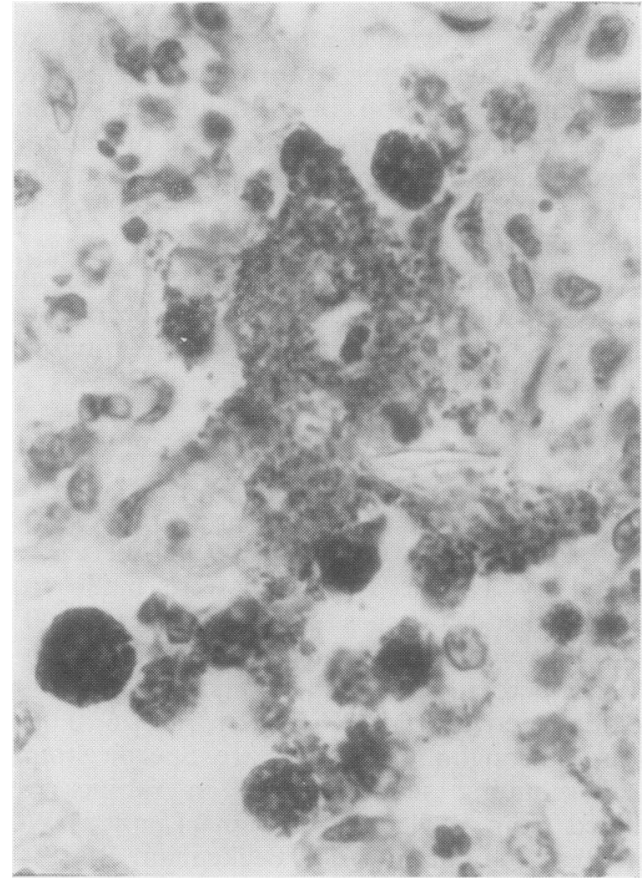

Fig. 1 (b) Polymorphonuclear leucocytes filled with dark staining cocci. Hyaline membrane also contains many cocci. ( $\times 1000$, PAP stain $)$ plexes of rabbit antiperoxidase and peroxidase (PAP) (DAKO-Immunoglobulin, Copenhagen, Denmark), prepared according to the method of Sternberger, ${ }^{9}$ were used in the procedure. Antibodies were diluted with Tris- $\mathrm{HCl}$ buffer. Optimum results were obtained with the following antibody dilutions: specific streptococcal grouping sera 1:300, swine anti-rabbit sera 1:20, and PAP reagent 1:100.

The tissue sections were exposed sequentially to each of the antisera. Antibody-bound peroxidase was demonstrated by the addition of diaminobenzidine $(0.6 \mathrm{mg} / 100 \mathrm{ml})$ in Tris- $\mathrm{HCl}$ buffer and $3 \%$ hydrogen peroxide. Sections were then counterstained with Harris haematoxylin, dehydrated through alcohols to xylol, and then permanently mounted.

\section{TESTS FOR SPECIFICITY}

In every case the PAP technique was employed, omitting the primary antisera to show that endogenous peroxidase activity was abolished. The primary antisera were absorbed with group B streptococci. The streptococci were fixed for 1 hour in $10 \%$ buffered formalin, concentrated by centrifugation, and then resuspended in the antisera to a concentration of three MacFarland's standards $\left(9 \times 10^{9}\right.$ organisms $\left./ \mathrm{ml}\right)$. Two overnight absorptions were performed. The absorbed sera were then tested on tissue sections known to contain group B 
streptococci. Specificity of the reaction was also tested by using the PAP method to stain air-dried bacterial suspensions using stock cultures of streptococci (groups A, B, F, G), viridans steptococci, Streptococcus pneumoniae, coagulase-positive and coagulase-negative staphylococci, and Escherichia coli. The bacterial suspensions were fixed for 1 hour in $10 \%$ buffered formalin. These suspensions were prepared at a concentration of one-half MacFarland's standard $\left(10^{8}\right.$ organisms $\left./ \mathrm{ml}\right)$, and $0.05 \mathrm{ml}$ of each suspension was air-dried on a microscopic slide before staining.

\section{Results}

\section{SPECIFICITY OF THE REACTION}

Stains of the air-dried bacterial suspensions prepared from stock cultures and fixed in $10 \%$ buffered formalin showed dark brown-black positive staining of bacteria only in the case of group B streptococci. In all cases treatment with methanol-peroxide abolished endogenous tissue peroxidase activity. Positive staining in tissue sections was abolished after absorption of the primary antisera with group B streptococci.

\section{RETROSPECTIVE NECROPSY STUDY}

Lung sections from three neonatal necropsies with pneumonitis and lung cultures positive for group B streptococci were studied. In each case dark brown-

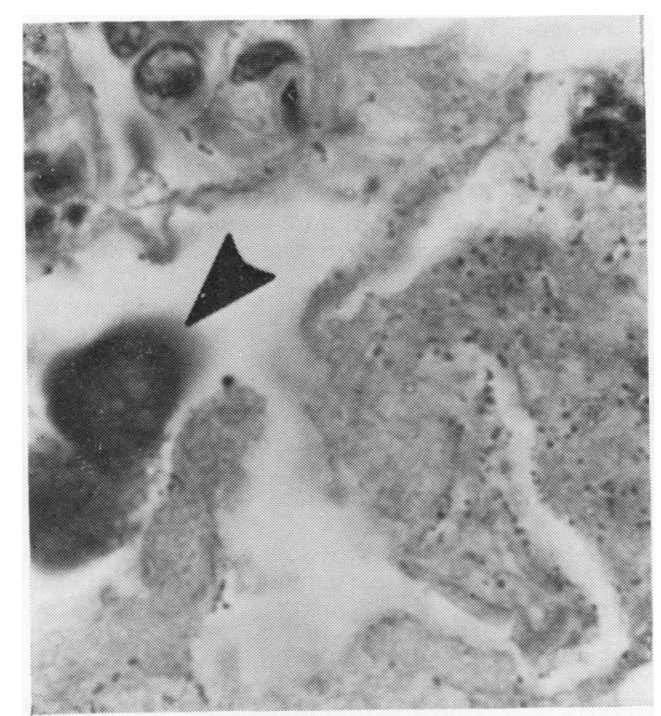

Fig. 2 Dark staining cocci were found within many hyaline membranes, and some hyaline membranes (arrow) showed diffuse dark staining, suggesting the presence of group B streptococcal specific antigen. ( $\times 1000$, P AP stain $)$

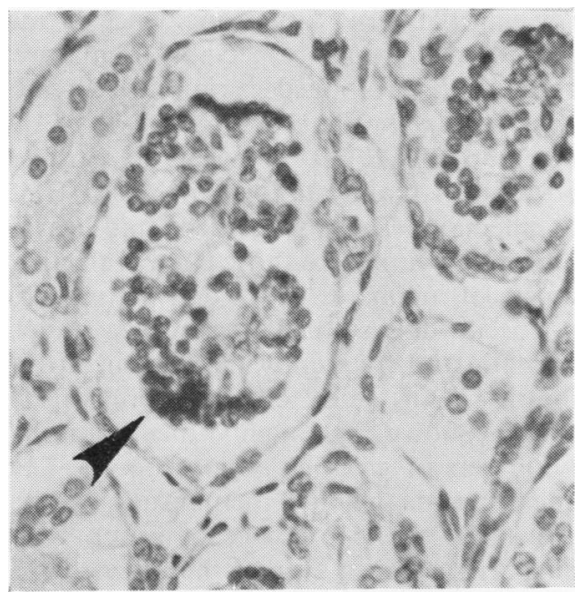

Fig. 3 Septic emboli were identified within multiple tissues of one case, including renal glomeruli. $(\times 450$, PAP stain)

black intra- and extra-cellular cocci were readily identified. Abundant microorganisms within leucocytes was a prominent feature (Fig. 1a, b). Compared to tissue Gram stains, organisms were identified more readily by the PAP stain. Dark staining intracellular organisms could be recognised at low light microscopic magnifications due to the pale background staining of the tissues. Intact cocci were found within many hyaline membranes. In addition, some hyaline membranes had distinct, diffuse, brown-black staining (Fig. 2). In one case, postmortem lung cultures were not obtained. The final diagnosis of group B streptococcal pneumonitis had been based on Gram-positive cocci in a tissue Gram stain of lung sections and the results of antemortem cultures. The PAP method confirmed the presence of group B streptococci. Finally, in a case of septicaemia due to group B streptococci, septic emboli were found in sections from multiple organs, including the lungs, kidneys, liver, and retroperitoneal nerves. The coccus was easily identified by the PAP technique (Fig. 3).

Eight additional neonatal deaths were studied in which group B streptococcal infection was not suspected. In these cases, hyaline membranes showed no staining, and no microorganisms were identified which had the staining characteristics of group B streptococci.

\section{Discussion}

The clinical features associated with group B streptococcal infection are well documented.1-311 These infants are usually premature and of low 
birth weight. There is a high incidence of maternal obstetric complications, especially premature onset of labour and prolonged rupture of membranes. The case fatality in the early-onset type of illness is greater than $50 \%{ }^{12}$ The cases we studied had typical clinical manifestations of group B streptococcal infection. The postmortem findings in these cases were also similar to those cited in the literature. Necropsy of neonates with early-onset group B streptococcal disease usually reveals evidence of pneumonitis and frequently the presence of hyaline membranes. Our study confirms previous reports which have shown that hyaline membranes contain large numbers of group B streptococci. ${ }^{11} 13$ Positive staining of some hyaline membranes by the PAP method suggests the presence of group B streptococcal specific antigen even in the absence of typical cocci. Katzenstein ${ }^{13}$ had noted this finding previously using the immunofluorescent technique.

The PAP technique described in this report may help in the postmortem evaluation of certain infectious diseases. By employing specific grouping serum, which is commercially available, this method can be used to confirm the presence of group B streptococci in routinely prepared histological sections. In this study the immunoperoxidase stain confirmed the results of lung cultures and tissue Gram stains. However, compared to Gram stains, microorganisms were found more readily using the PAP method. Previous reports have described the problems associated with postmortem microbiological investigations. ${ }^{4-8}$ The PAP method may help in necropsy cases where adequate cultures were not obtained or in cases where there is difficulty in interpreting results of cultures and tissue Gram stains. Additionally, this method could be used for the retrospective evaluation of postmortem material, as in the present study, or in selected cases seen at the surgical bench.

Other specific antisera are commercially available for bacteria with polysaccharide capsules. These include typing sera for Haemophilus influenzae type $b$, pneumococci, and meningococcus groups $\mathrm{A}, \mathrm{B}, \mathrm{C}$ and polyvalent groups $\mathrm{X}-\mathrm{Z}-\mathrm{W}$. Specific serotypes of group B streptococci in tissue sections might be identified utilising type specific antisera. In theory, each of these could be used as the primary antibody in the PAP technique. However, appropriate controls for specificity of the antibody must be rigidly observed in view of the extreme sensitivity of the PAP method. The PAP technique is a sensitive, reproducible method with great flexibility which has resulted in its increasing utilisation for a variety of purposes. $^{101415}$ As this method becomes more refined and 'routine' in the histological laboratory, multiple applications will be possible.

We gratefully acknowledge Professors JE Craighead, WC Winn, and TD Trainer for constructive criticism of the paper, and Mrs Joanne May for preparation of the text.

\section{References}

${ }^{1}$ Baker CJ. From the National Institutes of Health. Summary of the workshop on perinatal infections due to group B streptococcus. J Infect Dis 1977;136:137-52.

${ }^{2}$ Franciosi RA, Knostman JD, Zimmerman RA. Group B streptococcal neonatal and infant infections. $J$ Pediatr 1973;82:707-18.

${ }^{3}$ Quirante J, Ceballos R, Cassady G. Group B $\beta$-hemolytic streptococcal infection in the newborn. $A m J$ Dis Child 1974;128:659-65.

${ }^{4}$ Carpenter HM, Wilkins RM. Autopsy bacteriology: review of 2,033 cases. Arch Pathol Lab Med 1964; 77:73-81.

${ }^{5}$ Koneman EW, Minckler TM, Shires DB, deJongh DS. Postmortem bacteriology II. Selection of cases for culture. Am J Clin Pathol 1971;55:17-23.

${ }^{6}$ Koneman EW, Davis MA. Postmortem bacteriology III. Clinical significance of micro-organisms recovered at autopsy. Am J Clin Pathol 1974;61:28-40.

7 Wilson WR, Dolan CT, Washington JA, Brown AL, Ritts RE. Clinical significance of postmortem cultures. Arch Pathol Lab Med 1972;94:244-9.

${ }^{8}$ Dolan CT, Brown AL, Ritts RE. Microbiological examination of postmortem tissues. Arch Pathol Lab Med 1971 92:206-11.

${ }^{9}$ Sternberger LA. Immunocytochemistry. Edgewood Cliffs: Prentice-Hall, 1974.

10 Taylor CR. Immunoperoxidase techniques. Practical and theoretical aspects. Arch Pathol Lab Med 1978;102: 113-21.

${ }^{11}$ Ablow RC, Driscoll SG, Effman EL, Gross I, Jolles CJ, Uauy R, Warshaw JB. A comparison of early-onset group B streptococcal neonatal infection and the respiratory-distress syndrome of the newborn. $N$ Engl $J$ Med 1976;294:65-70.

${ }^{12}$ Baker CJ, Barrett FF. Group B streptococcal infections in infants; the importance of the various serotypes. $\mathrm{J} \mathrm{Am}$ Med Assoc 1974;230:1158-60.

${ }^{13}$ Katzenstein AL, Davis C, Braude A. Pulmonary changes in neonatal sepsis due to Group B $\beta$-hemolytic streptococcus: relation to hyaline membrane disease. $J$ Infect Dis 1976;133:430-5.

14 DeLellis RA, Sternberger LA, Mann RB, Banks PM, Nakane PK. Immunoperoxidase techniques in diagnostic pathology. Report of a workshop sponsored by the National Cancer Institute. Am J Clin Pathol 1979; $71: 483-8$.

15 Mesa-Tajada R, Pascal RR, Fenoglio CM. Immunoperoxidase: A sensitive immunohistochemical technique as a 'special stain' in the diagnostic 'pathology laboratory. Hum Pathol 1977;8:313-20.

Requests for reprints to: Dr TL Andres, Department of Pathology, The University of Vermont College of Medicine, Burlington, Vermont 05405, USA. 